\title{
Silver Nanocoatings at Large Length Scales: Influence of the AgNPs Morphology and Capping Agents on the Coating Chemical Stability and Antimicrobial Effect
}

\author{
Francisco A. Sousa, ${ }^{a, \#}$ Victor T. Noronha, ${ }^{a, \#}$ Terezinha F. Machado, ${ }^{b}$ José V. Silveira, \\ Francisco A. Cunha, ${ }^{d}$ Pierre B. A. Fechine ${ }^{d}$ and Amauri J. Paula ${ }^{*, a}$ \\ ${ }^{a}$ Grupo de Interfaces Sólido-Biológicas (SolBIN), Departamento de Física, \\ Universidade Federal do Ceará, P.O. Box 6030, 60455-900 Fortaleza-CE, Brazil \\ ${ }^{b}$ Embrapa Agroindústria Tropical, 60511-110 Fortaleza-CE, Brazil \\ ${ }^{c}$ Central Analítica and d Grupo de Química de Materiais Avançados (GQMAT), \\ Departamento de Química Analítica e Físico-Química, Universidade Federal do Ceará, \\ 60440-900 Fortaleza-CE, Brazil
}

\begin{abstract}
We assessed at multiple length scales (nanometers to millimeters) the nanocoatings of silver nanoparticles (AgNPs) on model $\mathrm{SiO}_{2} / \mathrm{Si}$ substrates. The coatings from biogenic AgNPs (from yeasts Rhodotorula glutinis and Rhodotorula mucilaginosa) were compared to those formed from "synthetic" AgNPs capped with citrate and sodium dodecyl sulfate (SDS). With computational analysis of large-field (LF) X-ray images of the whole substrates $(5 \times 5 \mathrm{~mm})$, we were able to assess the coatings homogeneity, relative amount of AgNPs, and their distribution as agglomerates. Surprisingly, by analyzing more than 100,000 elements (nanoparticles and agglomerates) in each sample, it was observed that the mentioned features have little dependence on the AgNPs morphology and capping agents. All silver nanocoatings resisted when immersed in phosphatebuffered saline medium by forming agglomerates of up to $10 \mu \mathrm{m}^{2}$. However, coatings formed with synthetic AgNPs (capped with citrate and SDS) led to a higher antimicrobial efficiency against Staphylococcus aureus.
\end{abstract}

Keywords: nanobiotechnology, image processing, confocal laser scanning microscopy, scanning electron microscopy, large-field X-ray imaging

\section{Introduction}

Silver nanoparticles (AgNPs) have many technological applications as films and coatings over solid surfaces such as metals, ceramic, glasses and polymers, to prevent growth of bacteria and fungi. ${ }^{1-5}$ For the AgNPs to be successfully attached, a suitable match between the solid surface and the nanoparticle physicochemical characteristics must occur. By synthesizing AgNPs with the mediation of bacteria and fungi extracts (biogenic AgNPs or bio-AgNPs), the resulting protein-stabilizing capping agent over the nanoparticle contains a variety of chemical groups bonded to the amino acids fragments. This latter aspect may lead to a myriad of possible chemical interactions (e.g. electrostatic, hydrophobic, hydrogen bonds and van der Waals) between the nanoparticles and solid surfaces. ${ }^{6-8}$

*e-mail: amaurijp@gmail.com

\#These authors contributed equally to this work.
Essentially, bio-AgNPs are formed from interaction mechanisms ruled by proteins present in the bacterial/ fungal extract or filtrate, which drive the bio-AgNPs' nucleation $\rightarrow$ growth $\rightarrow$ colloidal stabilization steps. ${ }^{2,9,10}$ At the end of the kinetic process, a protein corona phase on the bio-AgNPs provides a long-term colloidal stability. In contrast, "synthetic" AgNPs are produced from the reduction of $\mathrm{Ag}^{+}$and commonly stabilized by small capping agents of low molecular complexity, such as citrate and sodium dodecyl sulfate (SDS). ${ }^{11,12}$

Several insightful studies in the literature ${ }^{13-15}$ reported the formation of coatings and films of AgNPs (both biogenic and synthetic) and their dependence on the chemical functionalization of the solid surfaces upon which they are attached. However, the characterization of these coatings are limited to small length scales (up to microns), commonly achieved through imaging techniques such as electron microscopy performed at high magnifications. ${ }^{1,2,4,16-25}$ A complete understanding of the 
nanoparticle surface assembling requires a new imaging approach that provides information at all relevant length scales. The challenge is then to correlate the compositional, morphological, stereochemical and topographical features designed at the nanometer-scale (for both the solid surface and NPs), with the film/coating morphological and dimensional properties resulted from the NPs surfaceattachment at the micrometer to millimeter scales. Larger scales are particularly relevant for anti-fouling/anti-biofilm surfaces or materials designed for tissue regeneration. For example, in biofilms, bacterial cells initially bind to surfaces and form cluster at microscale levels. ${ }^{26}$ However, further bacterial cluster accumulation and biofilm growth occur at the upper-micron to millimeter scale. Therefore, enhanced knowledge of how AgNPs attach, assemble and stabilize at large length scales may be critical for understanding bacterial cell-biofilm growth mechanisms and prevention of infections that initiate with the microorganism attachment on solid surfaces. ${ }^{27-29} \mathrm{~A}$ similar premise can be applied for coatings to stimulate eukaryotic cell binding and tissue bioengineering, such as in bone regeneration.

To investigate the morphology/structure/dimensionality of the AgNPs coatings at multiple length scales (from nanometers to millimeters), we used large field (LF) X-ray imaging performed in a scanning electron microscope (SEM) ${ }^{30}$ The task of imaging at these multiple length scales can be performed by electron microscopy, mainly due to its high resolution at the bottom length scale (i.e. below $1 \mathrm{~nm}$ ). In addition, with the recent automatization of sample holders in SEM, sequential scanning of subjacent areas of a surface can be performed up to areas of centimeters. ${ }^{30} \mathrm{In}$ this way, quantitative information on the AgNPs distribution on the surface and the amount of nanoparticles per area was sought by processing the X-ray signal obtained from LF scans through image analysis algorithms. The differences in the coatings formed on model $\mathrm{SiO}_{2} / \mathrm{Si}$ substrates by synthetic and biogenic AgNPs, with varied morphologies (i.e. sizes and polydispersity) and surface functionalizations were investigated. Both the analytical approach and the results discussed here are provided in an attempt to fulfill an important gap of knowledge existing between the design of the nanometric entity at small length scales and their properties at large length scales (e.g. micrometers to centimeters), which are essentially attained from selfassembling mechanisms and interactions that dominate their organization and stability over a surface. By using this new imaging approach, we also intended to reveal the role of fundamental physicochemical interactions (e.g. electrostatic and van der Waals) on the AgNPs surfaceattachment and on the chemical stability of the resulting coatings.

\section{Experimental}

\section{Materials}

Silicon ( $\mathrm{Si}$ ) substrates were purchased from TedPella (USA). Potassium biphthalate/sodium hydroxide, disodium phosphate/monobasic potassium phosphate and boric acid/potassium chloride buffers were purchased from Neon Comercial Ltda (Brazil). Silver nitrate $\left(\mathrm{AgNO}_{3}\right)$ was purchased from Merck (Brazil). Glucose, sodium citrate and $\mathrm{NaCl}$ were purchased from Dinâmica (Brazil). Sodium dodecyl sulfate (SDS) was purchased from Vetec (Brazil). Malt extract, yeast extract, Mueller-Hinton medium agar, agar potato-glucose and peptone were purchased from Himedia (India).

\section{Microorganisms and biogenic silver nanoparticles (AgNPs)}

In this study we used yeasts Rhodotorula glutinis and Rhodotorula mucilaginosa isolated from soil samples collected at the Federal University of Ceará in the Pici campus $\left(3^{\circ} 44^{\prime} 20.832^{\prime \prime} S, 38^{\circ} 34^{\prime} 12.483^{\prime \prime} \mathrm{W}\right.$ for R. glutinis and $3^{\circ} 44^{\prime} 21.750^{\prime \prime} \mathrm{S}, 38^{\circ} 34^{\prime} 12.350^{\prime \prime} \mathrm{W}$ for R. mucilaginosa). An amount of $50 \mathrm{~g}$ of soil was transported to the laboratory in plastic bags conditioned in thermic boxes. The samples were then diluted in $\mathrm{NaCl}(0.9 \%)$, seeded in a medium containing malt extract $(0.3 \%)$, glucose $(1 \%)$, yeast extract $(0.3 \%)$, peptone $(0.5 \%)$ and antibiotics at $\mathrm{pH} 7$ (i.e. MGYP medium), and then incubated at $25^{\circ} \mathrm{C}$ for $72 \mathrm{~h}$. After growing, the samples were seeded in potato glucose agar and kept at $25^{\circ} \mathrm{C}$ for $72 \mathrm{~h}$. The yeasts that were identified as belonging to Rhodotorula gender were seeded again in potato glucose agar in order to obtain a pure and isolated strain. Genus identification was realized through a

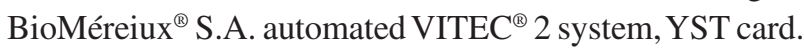

After isolating and identifying them, two yeast species were selected: $R$. glutinis and $R$. mucilaginosa. Yeasts were then grown in $500 \mathrm{~mL}$ Erlenmeyer flasks with $200 \mathrm{~mL}$ of MGYP medium at $25{ }^{\circ} \mathrm{C}$ for $72 \mathrm{~h}$. With the fungal biomass growth, the samples were centrifuged at $4000 \mathrm{rcf}$ for 10 minutes and washed three times for eliminating culture medium residues. Immediately after washing the yeasts were weighted (wet mass of $5.0 \mathrm{~g}$ ) and incubated in $100 \mathrm{~mL}$ of autoclaved deionized water for $48 \mathrm{~h}$ at $25^{\circ} \mathrm{C}$ (no stirring). The yeasts suspensions were then filtered in polyvinylidene difluoride (PVDF) membranes of $0.45 \mu \mathrm{m}$. Finally, to the suspension filtrate it was added $\mathrm{AgNO}_{3}$ in order to achieve $1.0 \mathrm{mmol} \mathrm{L}^{-1} \mathrm{of}_{\mathrm{Ag}^{+}}$concentration and the mixture was incubated at $25^{\circ} \mathrm{C}$ for $168 \mathrm{~h}$. Aliquots were periodically $(24 \mathrm{~h})$ drawn from the mixture in order to evaluate the AgNPs formation through UV-Vis spectrophotometry, in which the spectrum background 
(negative control) was obtained with the yeast filtrate containing $\mathrm{AgNO}_{3}\left(1.0 \mathrm{mmol} \mathrm{L}^{-1}\right)$. The confirmation of the AgNPs formation was attained with a characteristic absorption plasmonic band. ${ }^{31}$ The AgNPs purification after $168 \mathrm{~h}$ of incubation time was performed by centrifuging the mixture at $10000 \mathrm{rcf}$ for $20 \mathrm{~min}$. Pelletized AgNPs were washed three times with deionized water and finally resuspended in deionized water. For characterizations in which solid samples were required, the pellet was dried at room temperature instead of being resuspended. To handle with the AgNPs suspension produced from $R$. glutinis and R. mucilaginosa, the concentration of the nanoparticle suspension was considered as the total concentration of $\mathrm{Ag}^{+}$, determined by inductively coupled plasma spectrometry (ICP) after the AgNPs dissolution. It was used this concentration instead of considering just the nanoparticle concentration (calculated from thermogravimetric analysis), in order to consider the total amount of silver present in the suspension ( $\mathrm{Ag}$ and $\mathrm{Ag}^{+}$), thus avoiding experiments misinterpretations since $\mathrm{Ag}$ and $\mathrm{Ag}^{+}$coexist in equilibrium in the colloidal suspension, and both present biological effects. ${ }^{32-35}$

\section{Synthesis of AgNPs from glucose}

In order to compare the AgNPs coatings formed with different characteristics, they were produced from the reduction of glucose, and stabilized with sodium citrate and sodium dodecyl sulfate (SDS). Instead of the proteincapping present in the biogenic AgNPs produced from fungi R. glutinis and R. mucilaginosa, the so-called "synthetic" AgNPs present capping agents with smaller molecular complexity (i.e. citrate and SDS). The synthetic AgNPs (term used to differentiate them from the bio-AgNPs) were synthesized in $500 \mathrm{~mL}$ of a $5 \mathrm{mmol} \mathrm{L}^{-1}$ solution of silver nitrate $\left(\mathrm{AgNO}_{3}\right)$, containing $1.0 \mathrm{~g}$ of glucose as a reducing agent, and $0.5 \mathrm{~g}$ of the stabilizer (citrate or SDS). The $\mathrm{AgNO}_{3}$ solution was heated to $50{ }^{\circ} \mathrm{C}$ with a subsequent addition of the other reactants. The reaction was kept under magnetic stirring and it was catalyzed with the addition of $1.0 \mathrm{~mL}$ of $\mathrm{NaOH}\left(0.2 \mathrm{~mol} \mathrm{~L}^{-1}\right)$. After $1 \mathrm{~h}$ the solution got a yellowishbrownish color due to the formation of AgNPs. Nanoparticles were then centrifuged and washed three times with deionized water prior to be finally resuspended in deionized water. The AgNPs concentration in the suspensions was calculated in the same way as performed for bio-AgNPs (through ICP).

\section{Production of AgNPs coatings on $\mathrm{SiO}_{2} / \mathrm{Si}$ substrates}

Precut $5 \times 5 \mathrm{~mm}$ pieces of flat (root mean square roughness of $0.26 \mathrm{~nm}) \mathrm{Si}$ substrates $(<111>$ orientation;
$\mathrm{SiO}_{2}$ layer $<5 \mathrm{~nm}$ ) were cleaned by sonication in acetone for $5 \mathrm{~min}$ at high frequency $(40 \mathrm{kHz})$, and then thoroughly rinsed with deionized water produced by a Direct-Q ${ }^{\circledR} 3 \mathrm{UV}$ system (Millipore, USA). Over the cleaned silicon substrates, three sequential aliquots of $50 \mu \mathrm{L}$ of an AgNPs suspension (0.05 $\mathrm{mg} \mathrm{mL}^{-1}$ ) were carefully dropped, and the substrate was left to dry on a Petri dish over a heating plate at $50{ }^{\circ} \mathrm{C}$ between the sequential dropping steps. To unveil the possible influence of the AgNPs morphology and capping agents on the coating formation, the coatings were formed by using both biogenic and synthetic AgNPs (synthesized from glucose). After dropping the suspensions over the $\mathrm{SiO}_{2} / \mathrm{Si}$ substrates, chemically stable coatings were obtained by immersing the substrates in an upright position into $5 \mathrm{~mL}$ of a 1x-phosphate-buffered saline solution (PBS; $10 \mathrm{mmol} \mathrm{L}^{-1}$ of a phosphate buffer, $2.7 \mathrm{mmol} \mathrm{L}^{-1}$ of potassium chloride and $137 \mathrm{mmol} \mathrm{L}^{-1}$ of sodium chloride; $\mathrm{pH}$ 7.4). Most of the initial coating is lixiviated during this washing process, thus only tightly adhered AgNPs remained on the various substrates. After immersion in the PBS solution, AgNPs-coated Si substrates were kindly rinsed with $100 \mathrm{~mL}$ of ultrapure type 1 water in order to remove any salt present.

\section{Large-field X-ray imaging}

X-ray signal (acquired through energy dispersive spectroscopy; EDS) was captured along a large-field scan performed in the electron microscope Quanta-450 (FEI) with a field-emission gun (FEG), a $100 \mathrm{~mm}$ stage and an X-ray detector (model 150, Oxford). $\mathrm{SiO}_{2} / \mathrm{Si}$ substrates were introduced in the microscope chamber without sample preparation. Scans were performed in low vacuum mode (approximately $10 \mathrm{~Pa}$ with water vapor) for preventing sample charging. Micrographs and spectra were acquired at a beam acceleration voltage of $7 \mathrm{kV}$, and with a condenser aperture of $50 \mu \mathrm{m}$. For $7 \mathrm{kV}$ of acceleration voltage, the beam current over the specimen was of about $5 \mathrm{nA}$ (value provided by the manufacturer considering the conditions used in the column: condenser lens aperture, condenser lens convergence angle and accelerating voltage). To increase the beam high-vacuum path and minimize spurious beam skirting in the chamber (at low vacuum; $10 \mathrm{~Pa}$ ), a gaseous analytical cone (GAD) was used in all scans. The EDS detector was inserted at a collection angle of $55^{\circ}$ with the column axis, positioned approximately at the end of the polar piece. In all analyses, the working distance was set at approximately $12 \mathrm{~mm}$.

To generate the large-field images, an overlapping of marginal areas (a border which contains 20\% of the image area) was performed for adjacent images acquired independently after dislocations of the microscope stage 
along the $\mathrm{x}$ and $\mathrm{y}$ axes. The largest constructive interference between the two-dimensional distributions of grayscale values for the overlapped adjacent images determines the best positions for their placement. The resulting images, elemental maps and cumulative EDS spectra presented in this paper result from more than 300 adjacent electron beam scans acquired individually at $1500 \times$ magnification (horizontal and vertical fields of 0.274 and $0.188 \mathrm{~mm}$, respectively; $512 \times 352$ pixels). With this setup, the large-field scan took approximately $7 \mathrm{~h}$ for each substrate of $5 \times 5 \mathrm{~mm}$.

\section{Image processing}

In the elemental maps presented in the paper (Figures 1 and 2), just an inner area of $3.6 \times 3.6 \mathrm{~mm}$ of the full scanned substrate (an example is shown in Figure S4 in the Supplementary Information (SI) file) was used for assessment in order to avoid interpretation of artifacts introduced with the sample handling (performed always at the $\mathrm{SiO}_{2} / \mathrm{Si}$ substrate edge). The process used for cropping the $3.6 \times 3.6 \mathrm{~mm}$ area from the raw LF X-ray images
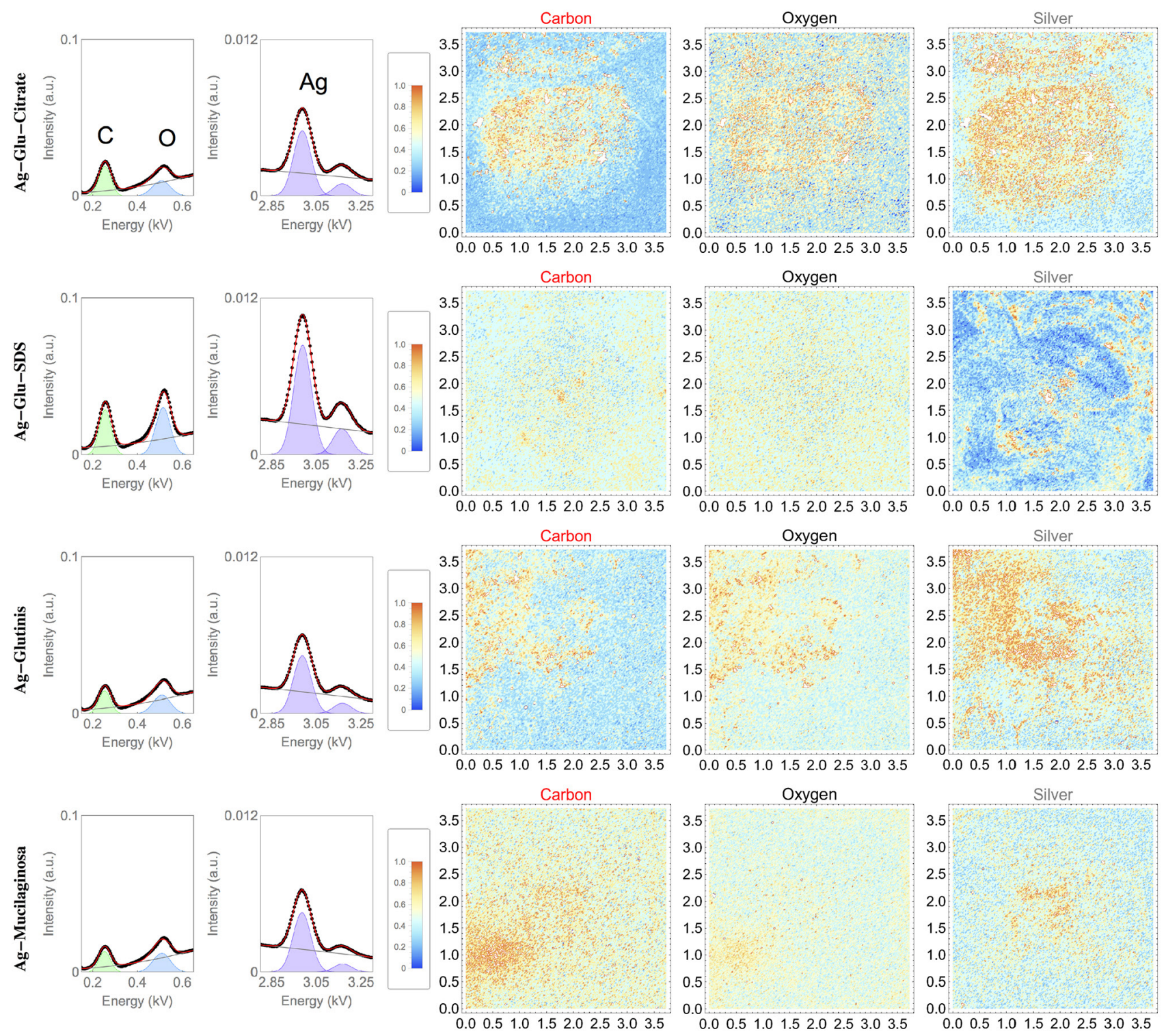

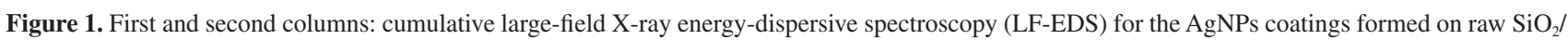
$\mathrm{Si}$ substrates. Coatings were formed from synthetic AgNPs produced from glucose and capped with citrate (Ag-Glu-Citrate) and sodium dodecyl sulfate (Ag-Glu-SDS), or from biogenic AgNPs produced with the extract of $R$. glutinis (Ag-Glutinis) and $R$. mucilaginosa (Ag-Mucilaginosa). The cumulative spectra shown in the first and second columns are the sum of hundreds of spectra individually obtained in a substrate area of ca. $2 \mu \mathrm{m}^{2}$. Analyses were performed on the substrates after a 6 hour-immersion washing step in a phosphate-buffered saline solution (PBS 1x). Elemental maps extracted from the LF-EDS data are depicted from third to fifth columns with approximately $3.6 \times 3.6 \mathrm{~mm}$. Each pixel in the maps is represented with a colored contrast function (from 0 to 1), which is a normalized scale given as a function of the element peak area (in the EDS spectrum) associated with that pixel. Maps scales are represented in millimeters. 

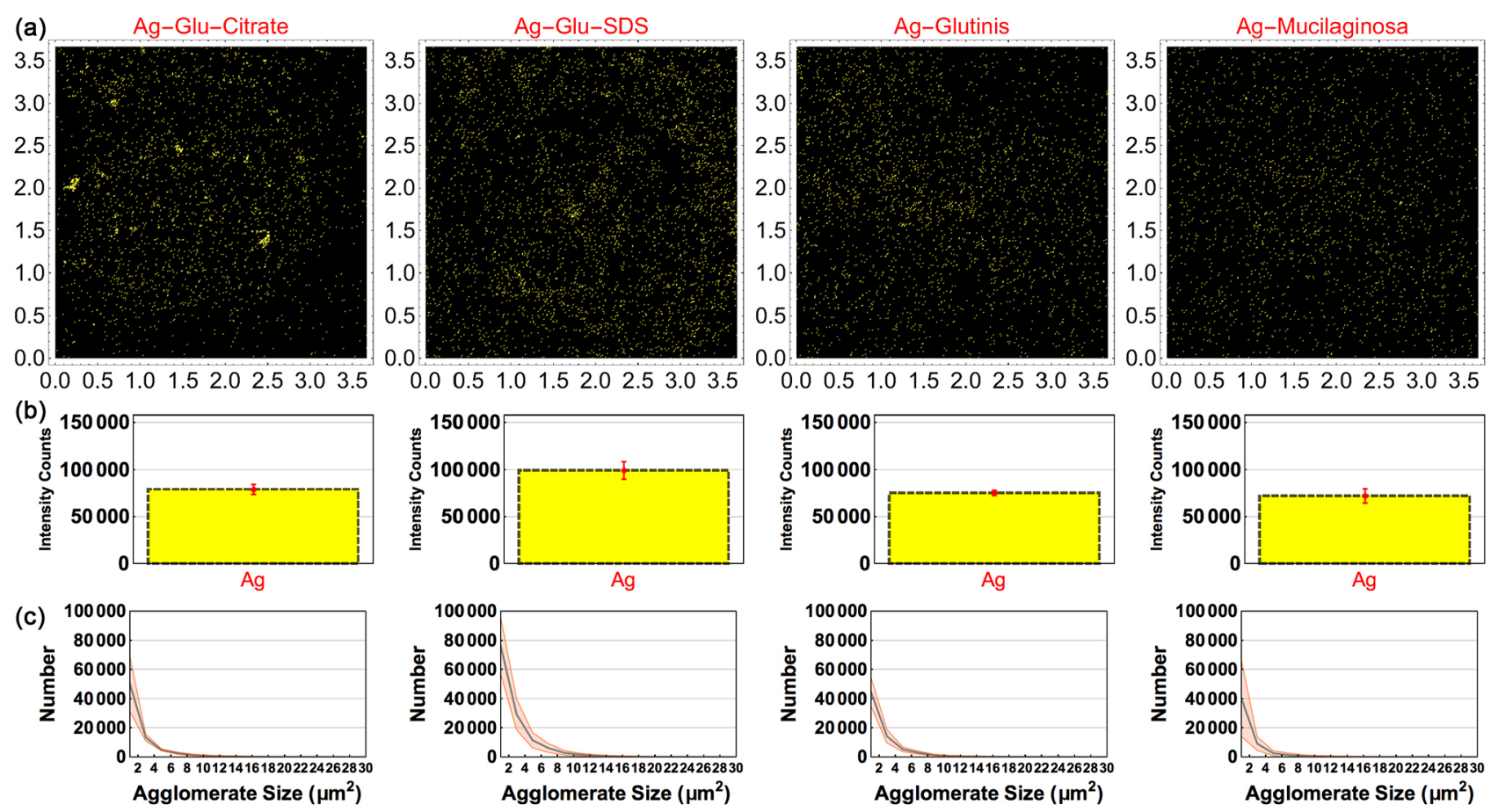

Figure 2. (a) Examples of maps used in the quantitative analysis of coatings formed on $\mathrm{SiO}_{2} / \mathrm{Si}$ substrates by using synthetic (Ag-Glu-Citrate and Ag-Glu-SDS) and biogenic (Ag-Glutinis and Ag-Mucilaginosa) silver nanoparticles. Graphs were obtained from the Ag large-field (LF) EDS elemental maps. Black pixels represent the absence of $\mathrm{Ag}$ and yellow pixels represent the presence of $\mathrm{Ag}$; (b) relative amount of $\mathrm{Ag}$ (given in intensity counts) calculated from the integration of the Ag EDS signal on the whole substrate area $(3.6 \times 3.6 \mathrm{~mm})$. Standard deviations were calculated from at least three LF scans performed for at least three independent substrates; (c) size distribution of the AgNPs agglomerates (given in area) on the surface. Shaded red area in the graphs represents the standard deviation while the gray curve represents the mean values.

(performed in Wolfram Mathematica) was systematically repeated for all samples. The process comprised $(i)$ an image rotation step to standardize the substrate position, (ii) an image crop step to exclude the border corresponding to the sample holder (i.e. exclusion of the lateral background), (iii) an image resize step to standardize the image definition (i.e. pixels per inch value), and (iv) an inner area crop step to exclude $0.7 \mathrm{~mm}$ from the borders of the substrate. The initial size of the raw LF images (both micrographs and elemental maps; see Figure S4 in the SI file) was of approximately $5000 \times 5000$ pixels ( 96 pixels per inch). After the binning and cropping processes, the resulting elemental maps used for the quantitative assessment of the AgNPs coatings (shown in Figures 1 and 2) had $2465 \times 2465$ pixels, with each pixel representing $1.4 \times 1.4 \mu \mathrm{m}$ of the substrate surface. Detailed information on the image processing steps used for obtaining the maps and calculations discussed in this paper is described in the SI file.

\section{Results and Discussion}

\section{Characterization of synthetic and biogenic AgNPs}

Yeasts resemble bacteria in some aspects, mainly if considered that they form isolated colonies and present a large enzymatic repertoire. However, there are fewer reports on their use for mediating the AgNPs production in comparison to those reporting on the use of bacterial extracts. ${ }^{36-39}$ Pigmented yeasts $R$. glutinis and $R$. mucilaginosa isolated in this study were observed to be able to reduce $\mathrm{Ag}^{+}$and to produce homogenous colloidal AgNPs from $\mathrm{AgNO}_{3}\left(1.0 \mathrm{mmol} \mathrm{L}{ }^{-1}\right)$ when this salt is dissolved in their fungal extract. The nanoparticle formation after $168 \mathrm{~h}$ was confirmed by its plasmon absorption band, manifesting at a wide range of the UV-Vis absorption spectrum (see Figure S1 in the SI file). In the specific case of the biogenic synthesis, it is difficult to interpret the evolution of the band shape due to the multiple kinetic processes occurring simultaneously, considering the myriad of biomolecules acting on the nanoparticle nucleation and growth. Even though, the maximum intensity of the band is a useful parameter to assess the nanoparticle concentration in the filtrate. We have stopped the reaction at $168 \mathrm{~h}$, time at which it was observed the most intense absorbance signal around $420 \mathrm{~nm}$ (absorbance was practically constant for further periods), in order to avoid the nanoparticle coarsening and preserve the colloidal features (i.e. size and polydispersity index). The resulting biogenic AgNPs formed both a characteristic brown-colored suspension. On the other hand, the production of synthetic AgNPs 
was performed by following well-established protocols reported in the literature that essentially involve the reduction of $\mathrm{Ag}^{+}$ions with a reducing agent and the use of a molecular capping agent to prevent particle aggregation and coalescence. ${ }^{40-42}$ By using glucose as the reducing agent, and citrate and sodium dodecyl sulfate as the capping agents, it was produced samples Ag-Glu-Citrate and Ag-Glu-SDS, respectively.

The morphology of both synthetic and biogenic AgNPs was analyzed by scanning electron microscopy (SEM) in high-vacuum mode and using secondary electrons (see Figure 3). All AgNPs produced presented a rounded morphology with particles of low aspect ratio (i.e. low anisotropy). The nanoparticle mean sizes determined in SEM images indicated that all AgNPs were sub-100 nanometer, with values smaller than $40 \mathrm{~nm}$, and standard deviations smaller than $23 \mathrm{~nm}$. Sample Ag-Glutinis had the smallest size $(18.1 \pm 6.5 \mathrm{~nm})$, while sample Ag-Glu-SDS had the broadest size distribution among the samples (see Figure S3 in the SI file). Another approach for comparing the variances of the AgNPs size distributions was performed for the nanoparticles in the colloidal state, which was made with dynamic light scattering (DLS) analyses of their suspensions in deionized water (see Figure S2 in the SI file). DLS results indicate that both biogenic AgNPs have a polydispersity index (PDI) of about 0.2 (see Table 1), although their average sizes were slightly different (108 nm for Ag-Glutinis and $120 \mathrm{~nm}$ for Ag-Mucilaginosa). The colloidal characteristics of the AgNPs generated by fungimediated processes recently reported largely differ from those obtained here in terms of polydispersity. It is currently known that the colloidal characteristics of the AgNPs depend on the $\mathrm{pH}$, temperature, and contact or incubation time during the synthesis. ${ }^{43}$ However, the kinetic processes involved with the nanoparticle nucleation and growth can be finely ruled by the pool of bio(macro)molecules present in the extract/filtrate used for their production, thus resulting in a specie-dependent biosynthesis. High-quality colloids of AgNPs (with small size and low polydispersity) can be biogenically produced depending on the species. ${ }^{44-47}$ On the other hand, the synthetic AgNPs produced had a more polydisperse character (i.e. larger variance in the size distribution), reflecting in a PDI of about 0.4 for both samples. In addition, their average sizes were of $170 \mathrm{~nm}$ for Ag-Glu-SDS and $190 \mathrm{~nm}$ for Ag-Glu-Citrate.

The larger size observed in DLS in comparison to the SEM micrographs is explained by the signal captured from the light scattered by the diffuse layers over the nanoparticle surface when they are dispersed in the liquid medium. This effect occurs mainly with the presence of the protein corona on biogenic AgNPs. On the other hand, in SEM images, the visualization of Ag metallic core is prioritized over the protein cap, since the proteins largely agglomerated during the drying step prior to the SEM analysis (see Figure 3).

In regard to the characteristics of the capping agents covering the AgNPs surface, zeta $(\zeta)$ potential analyses indicate that all samples are negatively charged at neutral pHs (used in the measurements). The exceptional colloidal stability observed for all samples in deionized water (stable for months at ca. $10^{\circ} \mathrm{C}$ ) is primarily a result of the high $\zeta$-potential values (below $-30 \mathrm{mV}$ for all samples; see Table 1). In the particular case of biogenic AgNPs (Ag-Glutinis and Ag-Mucilaginosa), the colloidal stabilization by steric/electrosteric mechanisms must also be accounted due to the presence of macromolecules in the protein corona. ${ }^{8,48}$

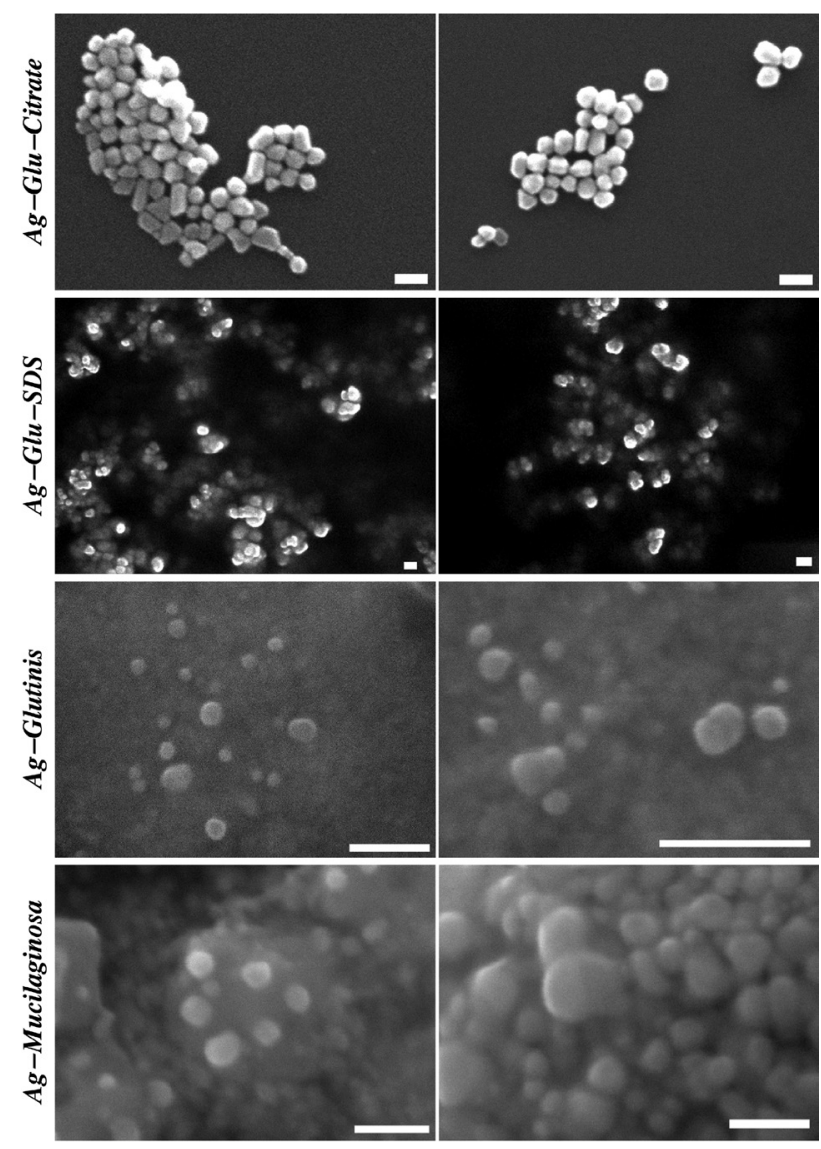

Figure 3. Scanning electron micrographs of synthetic AgNPs produced from glucose and capped with citrate (Ag-Glu-Citrate) and sodium dodecyl sulfate (Ag-Glu-SDS), and biogenic AgNPs produced from the extract of R. glutinis (Ag-Glutinis) and R. mucilaginosa (Ag-Mucilaginosa). White scale bars represent $100 \mathrm{~nm}$.

Assessment of the AgNPs coatings at multiple scales with LF X-ray imaging

The formation of the AgNPs coatings was assessed through EDS, acquired through a LF electron scanning. 
Table 1. Physicochemical characteristics of AgNPs

\begin{tabular}{|c|c|c|c|c|c|c|}
\hline \multirow{2}{*}{$\mathrm{AgNP}$} & \multicolumn{2}{|c|}{ DLS $^{\mathrm{a}}$} & \multicolumn{2}{|c|}{$\mathrm{SEM}^{\mathrm{c}}$} & \multicolumn{2}{|c|}{ Zeta potential $(\zeta)$} \\
\hline & Size / nm & $\mathrm{PDI}^{\mathrm{b}}$ & $\mathrm{Size}^{\mathrm{d}} / \mathrm{nm}$ & $\mathrm{STD}^{\mathrm{e}} / \mathrm{nm}$ & Value $^{\mathrm{f}} / \mathrm{mV}$ & $\mathrm{STD}^{\mathrm{e}} / \mathrm{mV}$ \\
\hline Ag-Glu-Citrate & 190 & 0.4 & 20 & 6.3 & -30 & 12 \\
\hline Ag-Glu-SDS & 170 & 0.4 & 34.5 & 22.1 & -41 & 17 \\
\hline Ag-Glutinis & 108 & 0.2 & 18.1 & 6.5 & -32 & 9 \\
\hline Ag-Mucilaginosa & 120 & 0.2 & 31.2 & 11.3 & -33 & 6 \\
\hline
\end{tabular}

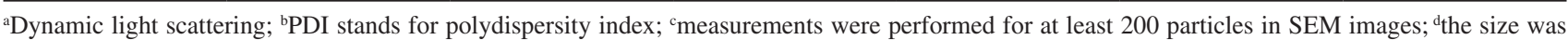
calculated by measuring the Feret Diameter in software Image J; ${ }^{\mathrm{e}} \mathrm{STD}$ : standard deviation; ${ }^{\mathrm{f}}$ measured with a nanoparticle suspension of $0.01 \mathrm{mg} \mathrm{mL}^{-1}$ in a $1.0 \mathrm{mmol} \mathrm{L}^{-1} \mathrm{KCl}$ solution.

Considering the $5 \times 5 \mathrm{~mm}$ silicon substrate, a square area of $3.6 \times 3.6 \mathrm{~mm}$ in the middle portion of the substrate was analyzed for all samples. Therefore, cumulative LF-EDS spectra shown in Figure 1 (first and second columns) correspond to the sum of all spectra obtained along the $3.6 \times 3.6 \mathrm{~mm}$ area of the substrate (more than 300 spectra). Well-resolved carbon (C $\mathrm{K} \alpha 0.27 \mathrm{keV})$ and oxygen peaks $(\mathrm{O} \mathrm{K} \alpha 0.52 \mathrm{keV})$ in the cumulative EDS spectra (see Figure 1, first column) are related to the presence of surface silanol $(\mathrm{Si}-\mathrm{OH})$ groups present at the surface of the $\mathrm{SiO}_{2}$ layer, as well as to the presence of capping agents in samples Ag-Glu-Citrate, Ag-Glu-SDS, Ag-Glutinis and Ag-Mucilaginosa. The presence of AgNPs that were firmly attached to the substrate after the immersion in PBS (for $6 \mathrm{~h}$ ) was evidenced with the $\mathrm{Ag}$ peak at about $3 \mathrm{keV}$ (L $\alpha$ emission; see Figure 1 second column). It is important to mention that the majority of AgNPs are lixiviated in this washing process.

The colored contrast function represented in the maps of Figure 1 (with values from 0 to 1 ) is a result of a normalization function applied for all elemental maps, through which the image pixel associated with the largest element peak area assumes a red color (i.e. largest peak area value in the EDS spectrum). For illustration, an example of fitting is applied for the cumulative LF-EDS spectra as shown in Figure 1 (first and second columns). A similar procedure was applied individually for all spectra present in each pixel of all elemental maps $(2465 \times 2465$ pixels). Absence of signal (i.e. EDS peak area value is zero) leads to the blue color. This colored contrast function facilitates the visualization of the elements (mainly those in low concentrations) in comparison with the conventional grayscale function, and makes possible a suitable comparison of the element distribution over the scanned area. By analyzing qualitatively the LF Ag maps (see Figure 1; last column), it was possible to observe that the AgNPs coatings covered the whole substrates at the micrometer scale. In addition, when the LF X-ray images of $\mathrm{C}, \mathrm{O}$ and $\mathrm{Ag}$ were compared, a large overlapping of the elemental maps was observed for all samples (Ag-Glu-Citrate, Ag-Glu-SDS, Ag-Glutinis and Ag-Mucilaginosa), thus indicating that the majority of $\mathrm{C}$ and $\mathrm{O}$ signals observed for the coatings are related to the AgNPs capping agents.

For the quantitative analysis of AgNPs coatings at the millimeter scale, the elemental maps from LF-EDS can provide better accuracy due to the small number of artifacts, which are essentially not observed in X-ray imaging at the length scales used here (see Figure S4, second column, in the SI file). In contrast, signal from electrons (both secondary and backscattered) emitted from the sample suffer charge interactions along the microscope chamber up to the detector, thus resulting in artifacts due to deflections and fluctuations in the signal processing. These artifacts become evident as seen through the "grid" pattern shown in Figure S4 (first column; see SI file). For determining (i) the homogeneity of the AgNPs coatings (Figure 2a), (ii) the concentration of AgNPs (Figure 2b), and (iii) the histograms of the AgNPs agglomerates sizes (Figure 2c), X-ray signal used for Ag had a spatial resolution of $1.4 \times 1.4 \mu \mathrm{m}$. Consequently, the primary $\mathrm{X}$-ray signal used in calculations (converted in the contrast value of a pixel in the elemental maps) was emitted from a $1.4 \times 1.4 \mu \mathrm{m}$ area of the surface (corresponding to 1 pixel in an image of $2465 \times 2465$ pixels). Comparatively, the coatings of sample Ag-Glu-Citrate and biogenic AgNPs (Ag-Glutinis and Ag-Mucilaginosa) that resisted in PBS had similar characteristics in regard to the relative amount of nanoparticles (70000-80000 intensity counts; see Figure $2 \mathrm{~b}$ ) present on the substrate. On the other hand, sample Ag-Glu-SDS had a slightly larger amount of AgNPs (see Figure 2).

The AgNPs coatings characterized by LF X-ray imaging were essentially comprised of nanoparticles agglomerates that ranged from hundreds of nanometers to tens of micrometers, distributed along the substrate. Interestingly, a similar size distribution function (ranging from 2 to $30 \mu \mathrm{m}^{2}$ ) was observed for the AgNPs agglomerates in all coatings, 
although sample Ag-Glu-SDS had a slightly larger amount of elements (see Figure 2c). These results were obtained by accounting more than 100,000 elements (AgNPs and agglomerates) identified in each LF Ag map (the smallest element had ca. $2 \mu \mathrm{m}^{2}$ ). This fact indicates that, regardless the nanoparticle morphology and capping agent, the coating chemical stability at this length scale is essentially attained with the formation of AgNPs agglomerates with specific sizes, of up to $10 \mu \mathrm{m}^{2}$ in area (along the substrate plane; see Figure 2c), and of a few hundreds of nanometers in height (see Figure 4). This is driven by nanoparticle desorption events that similarly manifested for attached AgNPs when the substrate was immersed in phosphate-buffered saline (PBS) solution. Furthermore, by calculating the mean distance (and standard deviation) between nearest agglomerates (i.e. neighbors) in samples Ag-Glu-Citrate, Ag-Glu-SDS, Ag-Glutinis and Ag-Mucilaginosa, it was found the values $6.6 \pm 3.2 \mu \mathrm{m}, 5.3 \pm 2.4 \mu \mathrm{m}, 5.9 \pm 2.2 \mu \mathrm{m}$, $6.0 \pm 2.9 \mu \mathrm{m}$. These values also indicate that the AgNPs distribution over the substrate was very similar for all samples tested.

The coating chemical stability on the $\mathrm{SiO}_{2} / \mathrm{Si}$ substrate when immersed in the PBS medium was attained with

SE
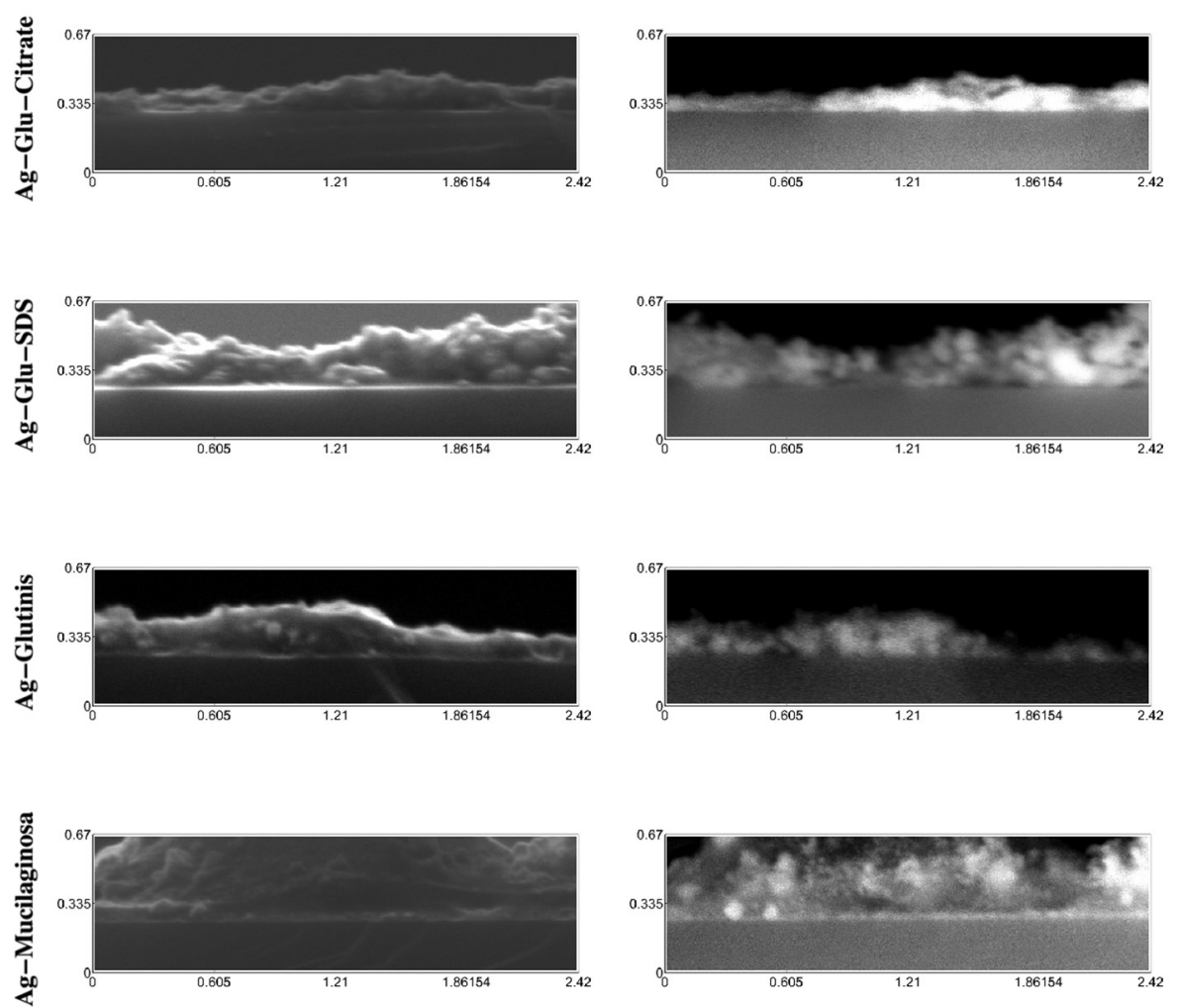

\section{BSE}

multiple attractive interactions occurring between AgNPs and the substrate. These interactions mainly include hydrogen bonds, which can specifically manifest from the AgNP capping agents interacting with the silanol ( $\mathrm{Si}-\mathrm{OH})$ groups on the $\mathrm{SiO}_{2} / \mathrm{Si}$ substrate surface (confirmed by $\mathrm{X}$-ray photoelectron spectroscopy; see Figure S5 in the SI file), and others of quantum-mechanical nature (e.g. van der Waals) which manifest at longer ranges. ${ }^{6-8,49}$ The attractive interactions (mainly van der Waals) may overcome possible electrostatic repulsion between the AgNPs and the substrate surface, since the latter had, to some extent, negatively charged groups covering the surface (by the deprotonation of silanol groups at neutral $\mathrm{pH})^{8,50,51}$ The predominance of these types of interactions over electrostatic repulsion has previously been observed for an adsorbate (i.e. peptide molecules) when its concentration is above a certain threshold. ${ }^{6}$ In this way, the stabilization of AgNPs as agglomerates played an important role for the coating to resist in the PBS medium (of high ionic strength), since the van der Waals attractive interactions manifested can be favored as a function of the AgNPs agglomerate size, defined by its area in the substrate plane and its height. 
In order to compare the anti-adherent and antimicrobial properties of the AgNPs coatings, the substrates were tested against a Staphylococcus aureus strain (see the SI file for more details). After the PBS washing process, the substrates were incubated with bacteria and then largefield confocal laser scanning microscopy (LF-CLSM) was used for determining the amount of live and dead bacteria attached to the substrates (see Figures S7 and S8a in the SI file). More than 10,000 elements (i.e. bacteria) were identified in LF-CLSM images for all samples, which enabled a precise assessment of the antimicrobial properties of the silver nanocoatings. In comparison with the raw $\mathrm{SiO}_{2} / \mathrm{Si}$ substrate, samples Si-Glu-Citrate, Si-GluSDS, Si-Glutinis and Si-Mucilaginosa had more bacteria attached. This fact is possibly related with the attachment sites created from the increase of the surface roughness with the silver nanocoating formation. Especially for coatings of biogenic AgNPs (mainly Si-Mucilaginosa), there is a soft carbonaceous matrix formed from the drying of the protein capping agent. As seen in Figure 4, biogenic AgNPs are embedded in a large carbon-based matrix formed from the biomolecules that act as surface caps. In regard to the relative efficiency of the silver nanocoatings, it was observed that coatings formed from synthetic AgNPs presented the lowest live/dead bacteria ratio, compared to coatings of biogenic AgNPs (see Figure S8b in the SI file). Overall, these findings reveal that, rather than the concentration of nanoparticles alone, it must be considered the influence of the capping agent on the antibacterial effect of nanocoatings (i.e. immobilized AgNPs on solid surfaces). Results here presented indicate that an excess of organic matter (from the capping agent) present in the resultant coating (after drying the AgNPs over the surface) may impair the AgNPs dissolution and $\mathrm{Ag}^{+}$diffusion towards bacterial cells and biofilms. Small capping agents such as citrate and sodium dodecyl sulfate led to the lowest live/dead ratio (highest efficiency; see Figure S8b). As previously reported, smaller $\mathrm{AgNPs}$ are easier to dissolve and release ions $\mathrm{Ag}^{+} .{ }^{52}$ In this study, this aspect would favor the antimicrobial activity of nanocoatings formed from biogenic AgNPs in comparison with those formed from synthetic ones (Ag-Glutinis and AgMucilaginosa had smaller nanoparticles). This is a fact that also supports the hypothesis that the carbonaceous matrix in which AgNPs are embedded in samples Ag-Glutinis and Ag-Mucilaginosa is impairing both the nanoparticle diffusion and their dissolution. Finally, it is important to mention that the AgNPs antimicrobial properties determined when the nanoparticle is suspended in a liquid (e.g. minimum inhibitory concentration (MIC), minimum bactericidal concentration (MBC)) can be very different from that observed when nanoparticles are immobilized and stabilized on a solid surface (e.g. anti-adherence, antifouling, anti-biofilm). In the latter context, an aspect that must be further investigated is how AgNPs dissolution, formation of passivation layers, re-deposition of silver and formation of bridging material between particles (e.g. organic matrix) influence the anti-fouling and anti-biofilm properties of silver nanocoatings at multiple length scales. ${ }^{52}$

\section{Conclusions}

The large-field (LF) X-ray imaging used here enabled us to compare the chemical stability of silver nanocoatings at large length scales (up to millimeters). Coatings formed from synthetic AgNPs capped with citrate or sodium dodecyl sulfate, and from biogenic AgNPs produced from yeasts $R$. glutinis and $R$. mucilaginosa could be quantitatively compared in regard to the amount of AgNPs covering the surface, as well as to the size distribution functions of their agglomerates. AgNPs from all sources and with different capping agents attached to $\mathrm{SiO}_{2} / \mathrm{Si}$ substrates and resisted to the immersion in phosphate-buffered saline (PBS; a medium of high ionic strength) with the formation of agglomerates of up to $10 \mu \mathrm{m}^{2}$. Interestingly, the resulting size distribution functions for the AgNPs agglomerates were very similar in all coatings assessed. This fact indicates that the mechanisms for generating such agglomerates in PBS (essentially through the AgNPs desorption) depended very little on the nanoparticle morphology and the surface capping agent. In addition, the amount of AgNPs identified in the coatings after immersing them in PBS was very similar for samples Ag-Glu-Citrate, Ag-Glutinis and AgMucilaginosa, while sample Ag-Glu-SDS had a slightly larger amount. However, in regard to their antibacterial properties, nanocoatings formed from synthetic AgNPs (capped with citrate and SDS) had a better efficiency against a strain of Staphylococcus aureus. This result may be associated with differences in the nanoparticle dissolution mechanisms and $\mathrm{Ag}^{+}$release from the coating, which can be limited by the soft matrix formed by the capping agents when the nanoparticles were dried on the surface, especially for biogenic AgNPs. Finally, despite the fact that in this study we focused on the study of the largescale surface assembly of AgNPs, the approach should be further applied for several nanoparticles of scientific and technological importance, in which there are properties (e.g. electric, magnetic, chemical, biological) that manifest at large length scales.

\section{Supplementary Information}

Supplementary data (complementary details on the 
characterization of AgNPs, image processing steps, UV-Vis absorption spectra, DLS measurements, LF images, XPS spectra, antibacterial assays and CLSM images) are available free of charge at http://jbcs.sbq.org.br as PDF file.

\section{Acknowledgments}

The authors are grateful for the support from the Brazilian Council for Scientific and Technological Development (CNPq), CAPES, FUNCAP and Central Analítica-UFC/ CT-INFRA/MCTI-SISNANO/Pró-Equipamentos-CAPES.

\section{References}

1. Durán, N.; Marcato, P. D.; de Souza, G. I. H.; Alves, O. L.; Esposito, E.; J. Biomed. Nanotechnol. 2007, 3, 203.

2. de Faria, A. F.; de Moraes, A. C. M.; Marcato, P. D.; Martinez, D. S. T.; Durán, N.; Filho, A. G. S.; Brandelli, A.; Alves, O. L.; J. Nanopart. Res. 2014, 16, 2110.

3. Mukherjee, P.; Ahmad, A.; Mandal, D.; Senapati, S.; Sainkar, S. R.; Khan, M. I.; Parishcha, R.; Ajaykumar, P. V.; Alam, M.; Kumar, R.; Sastry, M.; Nano Lett. 2001, 1, 515.

4. Zhang, M.; Zhang, K.; De Gusseme, B.; Verstraete, W.; Water Res. 2012, 46, 2077.

5. Durán, N.; Marcato, P. D.; Conti, R.; Alves, O. L.; Costa, F. T. M.; Brocchi, M.; J. Braz. Chem. Soc. 2010, 21, 949.

6. Puddu, V.; Perry, C. C.; ACS Nano 2012, 6, 6356.

7. Paula, A. J.; Araujo Júnior, R. T.; Martinez, D. S. T.; ParedesGamero, E. J.; Nader, H. B.; Durán, N.; Justo, G. Z.; Alves, O. L.; ACS Appl. Mater. Interfaces 2013, 5, 8387.

8. Paula, A. J.; Silveira, C. P.; Martinez, D. S. T.; Souza Filho, A. G.; Romero, F. V.; Fonseca, L. C.; Tasic, L.; Alves, O. L.; Durán, N.; ACS Appl. Mater. Interfaces 2014, 6, 3437.

9. Fayaz, A. M.; Balaji, K.; Girilal, M.; Yadav, R.; Kalaichelvan, P. T.; Venketesan, R.; Nanomedicine 2010, 6, 103.

10. Potara, M.; Bawaskar, M.; Simon, T.; Gaikwad, S.; Licarete, E.; Ingle, A.; Banciu, M.; Vulpoi, A.; Astilean, S.; Rai, M.; Colloids Surf., B 2015, 133, 296.

11. Ravindran, A.; Chandran, P.; Khan, S. S.; Colloids Surf., B 2013, $105,342$.

12. Wei, L.; Lu, J.; Xu, H.; Patel, A.; Chen, Z. S.; Chen, G.; Drug Discovery Today 2015, 20, 595.

13. Lv, Y.; Liu, H.; Wang, Z.; Liu, S.; Hao, L.; Sang, Y.; Liu, D.; Wang, J.; Boughton, R. I.; J. Membr. Sci. 2009, 331, 50.

14. Sidorov, A. N.; Sławiński, G. W.; Jayatissa, A. H.; Zamborini, F. P.; Sumanasekera, G. U.; Carbon N. Y. 2012, 50, 699.

15. Morrill, A. R.; Duong, D. T.; Lee, S. J.; Moskovits, M.; Chem. Phys. Lett. 2009, 473, 116.

16. Geng, X.; Qi, Z.; Li, M.; Duan, B. K.; Zhao, L.; Bohn, P. W.; Sol. Energy Mater. Sol. Cells 2012, 103, 98.
17. Enders, D.; Rupp, S.; Küller,A.; Pucci, A.; Surf. Sci. 2006, 600, 30.

18. Wei, M.; Wang, Y.; Materials 2015, 8, 3793.

19. Kyaw, H. H.; Al-harthi, S. H.; Sellai, A.; Dutta, J.; Beilstein J. Nanotechnol. 2015, 6, 2345.

20. Huo, S. J.; Xue, X. K.; Li, Q. X.; Xu, S. F.; Cai, W. B.; J. Phys. Chem. B 2006, 110, 25721.

21. Taglietti, A.; Arciola, C. R.; D’Agostino, A.; Dacarro, G.; Montanaro, L.; Campoccia, D.; Cucca, L.; Vercellino, M.; Poggi, A.; Pallavicini, P.; Visai, L.; Biomaterials 2014, 35, 1779.

22. Eby, D. M.; Luckarift, H. R.; Johnson, G. R.; ACS Appl. Mater. Interfaces 2009, 1, 1553.

23. Jo, Y. K.; Seo, J. H.; Choi, B.-H.; Kim, B. J.; Shin, H. H.; Hwang, B. H.; Cha, H. J.; ACS Appl. Mater. Interfaces 2014, 6, 20242.

24. Sileika, T. S.; Kim, H. D.; Maniak, P.; Messersmith, P. B.; ACS Appl. Mater. Interfaces 2011, 3, 4602.

25. Schneid, A. C.; Pereira, M. B.; Horowitz, F.; Mauler, R. S.; Matte, C. R.; Klein, M. P.; Hertz, P. F.; Costa, T. M. H.; Menezes, E. W.; Benvenutti, E. V.; J. Braz. Chem. Soc. 2015, 26, 1004.

26. Song, F.; Koo, H.; Ren, D.; J. Dent. Res. 2015, 94, 1027.

27. Thomas, W. E.; Curr. Opin. Struct. Biol. 2009, 19, 50.

28. Belas, R.; Trends Microbiol. 2014, 22, 517.

29. Kolewe, K. W.; Peyton, S. R.; Schiffman, J. D.; ACS Appl. Mater. Interfaces 2015, 7, 19562.

30. Oliveira, N. C.; Silva, J. H.; Barros, O. A.; Pinheiro, A. P.; Santana, W.; Saraiva, A. A. F.; Ferreira, O. P.; Freire, P. T. C.; Paula, A. J.; Anal. Chem. 2015, 87, 10088.

31. Gaikwad, S. C.; Birla, S. S.; Ingle, A. P.; Gade, A. K.; Marcato, P. D.; Rai, M.; Durán, N.; J. Braz. Chem. Soc. 2013, 24, 1974.

32. Zhao, C. M.; Wang, W. X.; Environ. Toxicol. Chem. 2011, 30, 885.

33. Foldbjerg, R.; Olesen, P.; Hougaard, M.; Dang, D. A.; Hoffmann, H. J.; Autrup, H.; Toxicol. Lett. 2009, 190, 156.

34. Kang, F.; Alvarez, P. J. J.; Zhu, D.; Environ. Sci. Technol. 2014, $48,316$.

35. Hadioui, M.; Leclerc, S.; Wilkinson, K. J.; Talanta 2013, 105, 15.

36. Kaler, A.; Jain, S.; Banerjee, U. C.; Biomed Res. Int. 2013, 2013, DOI: $10.1155 / 2013 / 872940$.

37. Mohanpuria, P.; Rana, N. K.; Yadav, S. K.; J. Nanopart. Res. 2008, 10, 507.

38. Apte, M.; Sambre, D.; Gaikawad, S.; Joshi, S.; Bankar, A.; Kumar, A. R.; Zinjarde, S.; AMB Express 2013, 3, 32.

39. Thakkar, K. N.; Mhatre, S. S.; Parikh, R. Y.; Nanomedicine 2010, 6, 257.

40. Mallmann, E. J. J.; Cunha, F. A.; Castro, B. N. M. F.; Maciel, A. M.; Menezes, E. A.; Fechine, P. B. A.; Rev. Inst. Med. Trop. S. Paulo 2015, 57, 165.

41. Henglein, A.; Giersig, M.; J. Phys. Chem. B 1999, 103, 9533.

42. Kvítek, L.; Panácek, A.; Soukupova, J.; Kolar, M.; Vecerova, R.; Prucek, R.; Holecova, M.; Zboril, R.; J. Phys. Chem. C 2008, 112,5825 . 
43. Akhtar, M. S.; Panwar, J.; Yun, Y.-S.; ACS Sustainable Chem. Eng. 2013, 1, 591.

44. Chan, Y. S.; Mat Don, M.; Mater. Sci. Eng., C 2013, 33, 282.

45. Madden, O.; Naughton, M. D.; Moane, S.; Murray, P. G.; Adv. Colloid Interface Sci. 2015, 225, 37.

46. Du, L.; Xu, Q.; Huang, M.; Xian, L.; Feng, J. X.; Mater. Chem. Phys. 2015, 160, 40.

47. Jain, N.; Bhargava, A.; Majumdar, S.; Tarafdar, J. C.; Panwar, J.; Nanoscale 2011, 3, 635.

48. Padovani, G. C.; Petry, R.; Holanda, C. A.; Sousa, F. A.; Saboia, V. M.; Silva, C. A.; Paschoal, A. R.; Souza Filho, A. G.; Paula, A. J.; J. Phys. Chem. C 2015, 119, 18741.
49. Roach, P.; Farrar, D.; Perry, C. C.; J. Am. Chem. Soc. 2005, 127, 8168.

50. Leung, K.; Nielsen, I. M. B.; Criscenti, L. J.; J. Am. Chem. Soc. 2009, 131, 18358.

51. Paula, A. J.; Montoro, L. A.; Filho, A. G. S.; Alves, O. L.; Chem. Commun. 2012, 48, 591.

52. Le Ouay, B.; Stellacci, F.; Nano Today 2015, 10, 339.

Submitted: September 8, 2016 Published online: November 29, 2016 